\title{
Green Radio: Radio Techniques to Enable Energy-Efficient Wireless Networks
}

\author{
Congzheng Han, Tim Harrold, and Simon Armour, University of Bristol \\ Ioannis Krikidis, Stefan Videv, Peter M. Grant, Harald Haas, and John S. Thompson, University of Edinburgh \\ Ivan Ku and Cheng-Xiang Wang, Heriot-Watt University
}

Tuan Anh Le and M. Reza Nakhai, Kings College London

Jiayi Zhang and Lajos Hanzo, University of Southampton

\begin{abstract}
Recent analysis by manufacturers and network operators has shown that current wireless networks are not very energy efficient, particularly the base stations by which terminals access services from the network. In response to this observation the Mobile Virtual Centre of Excellence (VCE) Green Radio project was established in 2009 to establish how significant energy savings may be obtained in future wireless systems. This article discusses the technical background to the project and discusses models of current energy consumption in base station devices. It also describes some of the most promising research directions in reducing the energy consumption of future base stations.
\end{abstract}

\section{INTRODUCTION}

Given the worldwide growth in the number of mobile subscribers, the move to higher-data-rate mobile broadband, and the increasing contribution of information technology to the overall energy consumption of the world, there is a need on environmental grounds to reduce the energy requirements of radio access networks. A typical mobile phone network in the United Kingdom may consume approximately 40-50 MW, even excluding the power consumed by users' handsets. In developing countries direct electricity connections are not readily available, so Vodafone, for example, use in excess of 1 million gallons of diesel per day to power their network. Mobile communications thus contributes a significant proportion of the total energy consumed by the information technology industry.

From an operator's perspective, reducing energy consumption will also translate to lower operating expenditure (OPEX) costs. Reducing carbon emissions and OPEX for wireless cellular networks are two key reasons behind the development of the Mobile VCE Green Radio program.
For example, the U.K. operators Orange and Vodafone both aim to achieve significant reductions in $\mathrm{CO}_{2}$ emissions in the next 10 years. The Green Radio program sets the aspiration of achieving a hundredfold reduction in power consumption over current designs for wireless communication networks. This challenge is rendered nontrivial by the requirement to achieve this reduction without significantly compromising the quality of service (QoS) experienced by the network's users. In order to meaningfully measure success, appropriate measures of energy consumption must be applied. For example, a reduction in radiated power is not of benefit if it is achieved at the expense of a greater increase in power consumed in signal processing or vice versa.

The Green Radio project is pursuing energy reduction from two different perspectives. The first is to examine alternatives to the existing cellular network structures to reduce energy consumption. The second approach, discussed in detail in the present article, is to study novel techniques that can be used in base stations or handsets to reduce energy consumption in the network. We present the background to the project. We move on to discuss base station modeling, which is a critical issue for the project. We then present three case studies that describe the energy savings obtainable from different techniques that can be employed on wireless links. Finally, we present conclusions to the article.

\section{REDUCING ENERgy CONSUMPTION IN WIRELESS NETWORKS}

The specific objective of the Green Radio program is to investigate and create innovative methods for the reduction of the total energy needed to operate a radio access network and to identify appropriate radio architectures that enable such a power reduction. The typical power consumption of different elements of a 


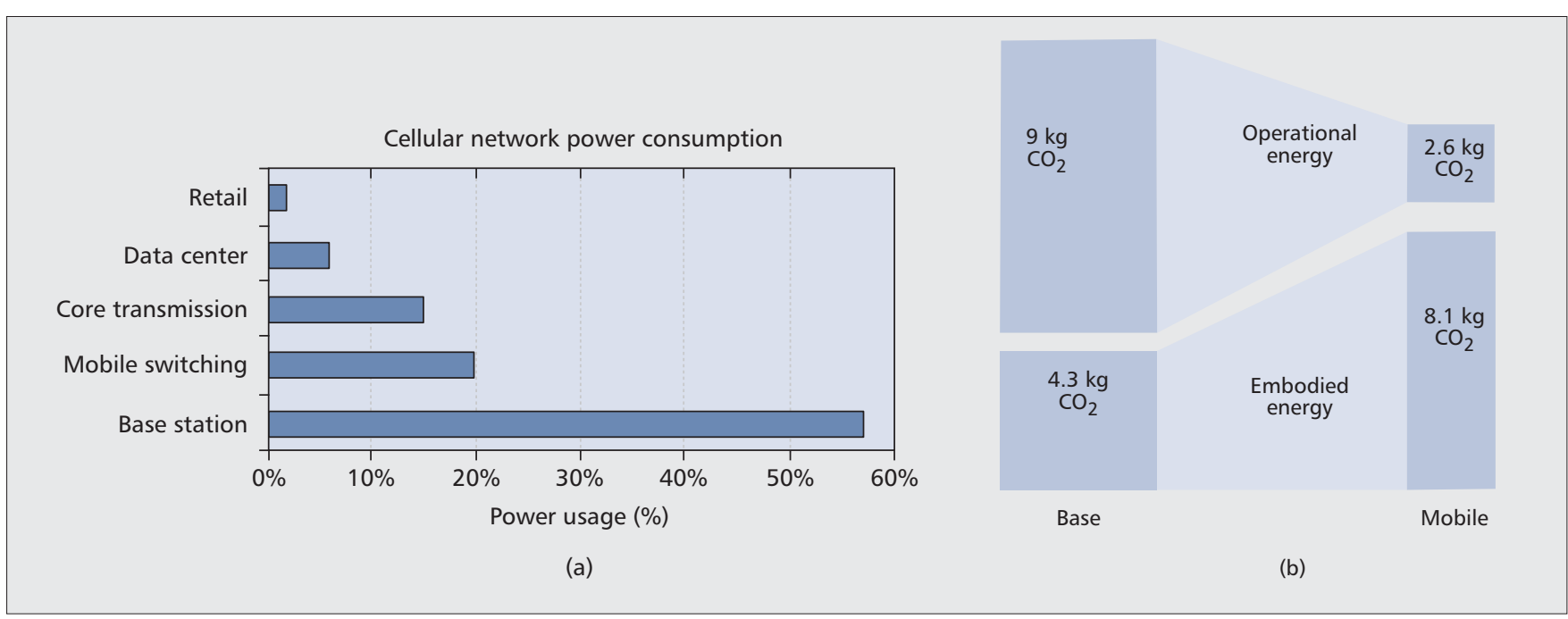

Figure 1. a) Power consumption of a typical wireless cellular network (source: Vodafone); b) $\mathrm{CO}_{2}$ emissions per subscriber per year as derived for the base station and mobile handset, after [1]. Embodied emissions arise from the manufacturing process rather than operation.

current wireless network is shown in Fig. 1a. These results clearly show that reducing the power consumption of the base station or access point has to be an important element of this research program.

Studies have indicated that the mobile handset power drain per subscriber is much lower than the base station component, Fig. 1b [1]; hence, the Green Radio project will mainly focus on base station design issues. Figure 1b also shows that the manufacturing or embodied energy is a much larger component in the mobile handset than in the base station. This is because the lifetime of a base station is typically 10-15 years, compared to a typical handset being used for 2 years. In addition, the energy costs of a base station are shared between many mobile subscribers, leading to a large imbalance in the contribution of embodied energy. From the point of view of handsets, significant efforts need to be put into reducing manufacturing energy costs and increasing handset lifetime, through recycling programs, for example. The Third Generation Partnership Project (3GPP) Long Term Evolution (LTE) system has been chosen as the baseline technology for the research program; its specifications have recently been completed with a view to rolling out networks in the next two to three years [2].

The next section of this article discusses the architecture of existing base stations and identifies key parts of the system hardware where significant energy savings can be obtained.

\section{Base Station POWER EfFiciency STUdies}

The overall efficiency of the base station, in terms of the power drawn from its supply in relation to its radio frequency (RF) power output, is governed by the power consumption of its various constituent parts, including the core radio devices.

Radio transceivers: The equipment for gener- ating transmit signals to and decoding signals from mobile terminals.

Power amplifiers: These devices amplify the transmit signals from the transceiver to a high enough power level for transmission, typically around 5-10 W.

Transmit antennas: The antennas are responsible for physically radiating the signals, and are typically highly directional to deliver the signal to users without radiating the signal into the ground or sky.

Base stations also contain other ancillary equipment, providing facilities such as connection to the service provider's network and climate control. A major opportunity to achieve the power reduction targets of the program lies in developing techniques to improve the efficiency of base station hardware.

Analysis within the program has developed models for various base station configurations (macrocell, microcell, picocell, and femtocell) in order to establish how improvements in the hardware components will impact the overall base station efficiency. The starting point for this analysis has been the transmit chain. Near-market power consumption figures have been used in order to establish a benchmark efficiency against which improvements made as part of the project can be assessed. Target power consumption figures allow future overall base station efficiencies to be predicted.

\section{Reference Base Station Architecture}

The target system for the base station efficiency analysis is the LTE system with support for four transmit antennas. This system can exploit the space domain to achieve high data throughputs through multiple input multiple output (MIMO) techniques [2]. The reference architecture under investigation is shown in Fig. 2, this represents a macrocellular base station with three sectors, with an effective isotropic radiated power (EIRP) of $27 \mathrm{dBW}$ per sector. The four transmit chains needed for the four antennas therefore require 12 power amplifiers (PAs) and antennas 


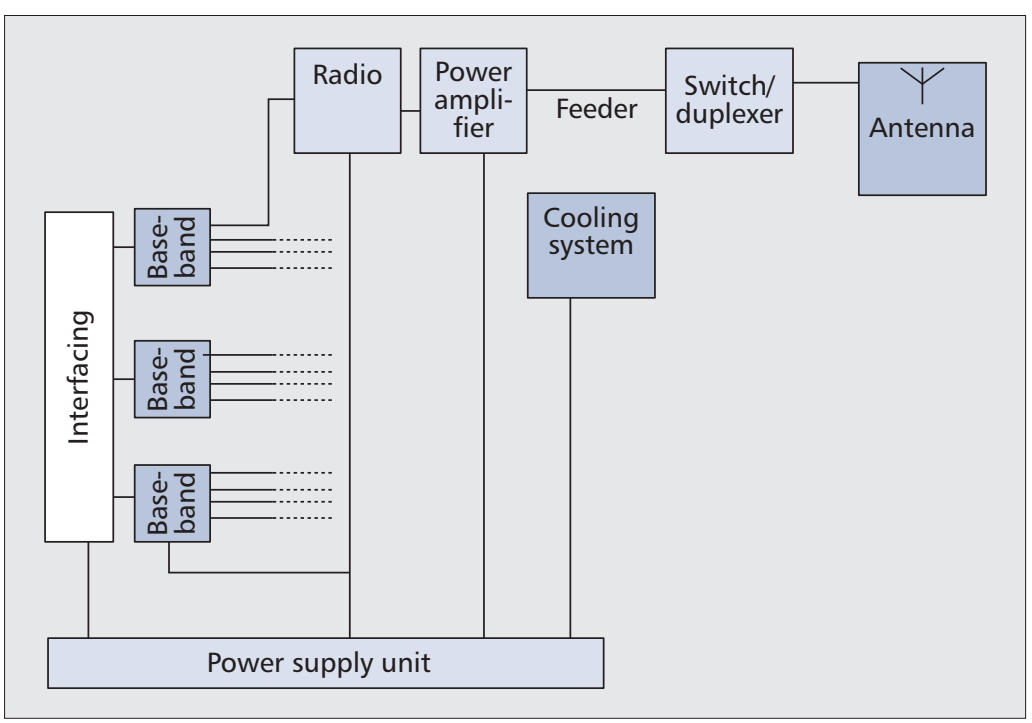

Figure 2. Reference base station architecture for a system with three sectors and four transmit antennas per sector for MIMO capability. For clarity only one transmit chain is shown. for increasing the overall base station efficiency comes from improving the efficiency of the PA and antenna, as well as optimizing the power transfer between them. Work underway in the program is seeking to achieve efficiency figures of 85 and 90 percent for these components, respectively. In the case of the PA, one possible approach uses the Class J amplifier [5], which relies on fundamental and second harmonic tuning to achieve high efficiencies, while maintaining the linearity required for LTE operation. In the case of the antenna, the 90 percent efficiency target is to be achieved by exploiting highly efficient dual-polarized patch antenna elements.

\section{CASE STUDIES FOR IMPROVING ENERGY EFFICIENCY IN Wireless Base Stations}

Earlier the power consumption of base stations was discussed and strategies to minimize power use in future base stations was described. In this section, we will move on to consider approaches which are designed around the signals that are transmitted by the base stations. In this case, the time dimension of these waveforms becomes important. In such a case, measures of energy (power $\times$ time) rather than just power become important metrics to measure system performance effectively. This section will therefore begin by discussing suitable energy metrics and then move on to discuss three case studies, based around resource allocation, interference cancellation, and the use of multihop relaying strategies.

\section{OVerview of Energy Metrics}

The results in Fig. 1a of this article show the fact that base stations account for a significant proportion of the total power consumption of a wireless network. If new techniques are proposed to reduce the energy required in the network, it is important to provide meaningful metrics that identify what gains are achieved. The metrics to be used in the Green Radio project have been discussed extensively, and there are two particularly important metrics that are intended to be used during the project.

The first is an absolute measure of energy and is closely related to the industry concept of the energy consumption rating (ECR). This is typically defined as a ratio of peak power divided by the maximum data throughput for a base station transmitter. However, to be of practical use, the ECR should measure the consumed energy per information bit that is successfully transported over the network and is measured in units of joules per bit. This metric allows the absolute performance of different wireless networks to be calibrated. As a simple example, a typical LTE base station sector might operate over a bandwidth of $10 \mathrm{MHz}$ with an average spectral efficiency of $1.5 \mathrm{~b} / \mathrm{s} / \mathrm{Hz}$, thus achieving an average data rate of $15 \mathrm{Mb} / \mathrm{s}$. If a base station antenna transmits $8 \mathrm{~W}$ of RF power (Table 1), the RF ECR value for this system would be 0.53 $\mu \mathrm{J} / \mathrm{b}$. However, if the total power budget of the 


\begin{tabular}{|c|c|c|c|c|}
\hline Description & Power In (W) & Power Out (W) & Efficiency & Target Value \\
\hline Radiated power (per sector) & 8 & $501(27 d B W)$ & $\begin{array}{l}18 \mathrm{dBi} \text { antenna } \\
\text { gain }\end{array}$ & $\begin{array}{l}18 \mathrm{dBi} \text { antenna } \\
\text { gain }\end{array}$ \\
\hline Antenna and Switch & 12 & 8 & $65 \%$ efficient & $85 \%$ efficient \\
\hline Feeder & 24 & 12 & $50 \%$ efficient & $80 \%$ efficient \\
\hline PA (total per sector) & 60 & 24 & $40 \%$ efficient & $85 \%$ efficient \\
\hline PA (all sectors) & 180 & 72 & & \\
\hline Transceiver (all sectors) & 180 & & & $70 \%$ reduction \\
\hline Free Air Cooling & 40 & & & \\
\hline Subtotal & 400 & & & \\
\hline PSU Input & 450 & 400 & $88 \%$ efficient & $88 \%$ efficient \\
\hline TOC Efficiency & & & $16 \%$ & $>25 \%$ \\
\hline Radiated Efficiency & & & $5.3 \%$ & $>20 \%$ \\
\hline
\end{tabular}

Resource allocation

techniques that

make the most effi-

cient use of the $R F$

amplifier have the

potential to improve

energy efficiency sig-

nificantly. Such ener-

gy reductions could

lead to further ener-

gy savings through

switching off

transceiver equip-

ment and base sta-

tion cooling.

Table 1. Estimated power consumption for base stations in 2010-2011 and target future power consumption values for base stations.

base station (e.g., $450 \mathrm{~W}$ ) is shared among 3 sectors (i.e., $150 \mathrm{~W} /$ sector) the ECR value for one sector would increase to $10 \mu \mathrm{J} / \mathrm{b}$.

The second metric is a relative measure rather than an absolute one and is more useful for comparing two different systems. Frequently, one may wish to compare the energy performance of a base station using a newly proposed technique (system under test) and compare to a baseline system where the approach is not deployed. The energy consumption gain (ECG) is simply the ratio $\left(E_{b} / E_{t}\right)$, where $E_{b}$ is the energy consumed by the baseline system and $E_{t}$ is the energy for the system under test. The larger the value of the ECG, the more efficient the system under test becomes. However, as with the ECR metric, care needs to be taken to ensure that the energy calculations are performed in a fair manner. For example, if two base station designs are being compared, it should be ensured that both are serving the same number of users under the same traffic load conditions, in order to provide a fair comparison.

\section{CASE Study 1: \\ Resource Allocation Strategies}

RF amplifiers were identified as a key contributor to the overall energy consumption of a typical base station. In this article we use the term resource allocation to describe how the base station transmitter make the decision of how and when to transmit data to different users on the downlink (base-mobile link) within the cell it is serving. Resource allocation techniques that make the most efficient use of the RF amplifier have the potential to improve energy efficiency significantly. Such energy reductions could lead to further energy savings through switching off transceiver equipment and base station cooling.
In addition, analysis of data traffic in wireless networks show that the traffic load is typically very uneven across the cells. In the analysis of 200 cells in [2, Ch. 9], it is shown that even in peak hours, 90 percent of the data traffic is carried by only 40 percent of the cells in the network. Therefore, techniques that minimize energy consumption across varying traffic load conditions are an important research direction; here we describe two complementary techniques aimed at low and high traffic load conditions, respectively.

Under low traffic load conditions, the base station is likely to have more bandwidth available to transmit data to users than is actually required at that time. One frequency domain approach being studied in the project exploits spare bandwidth resources to reduce energy consumption. Due to the fact that channel capacity scales linearly with the available bandwidth but logarithmically with the radio transmission power, it is possible to trade spectral for energy efficiency, and achieve energy savings while retaining quality of service [6]. Rather than use a complex but spectrally efficient modulation scheme (e.g., 16-quadrature amplitude modulation [QAM]) with a narrow bandwidth, it is possible to use a simpler modulation scheme (e.g., quaternary phase shift keying [QPSK]) with a wider bandwidth.

Figure 3a shows predicted ECG gain results for this approach, as a function for the signal-tointerference-plus-noise ratio (SINR) required at the mobile receiver for a given data rate. Generally speaking, as the spectral efficiency of the data rate increases, so does the required SINR. The value of $\alpha$ specifies the permitted bandwidth expansion factor, and curves are shown for values of $\alpha$ in the range 2-6. For example, a bandwidth expansion of $\alpha=2$ would permit 16- 


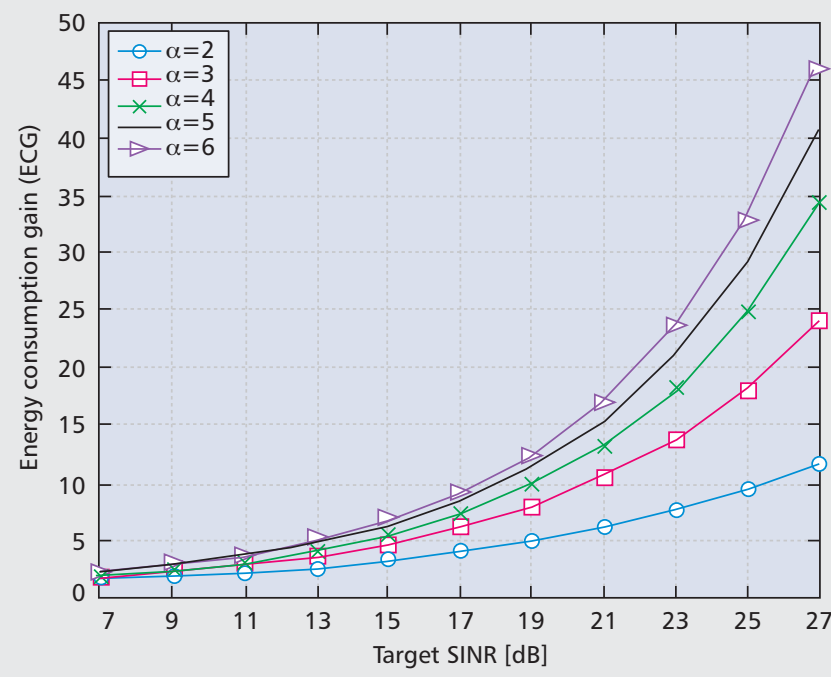

(a)

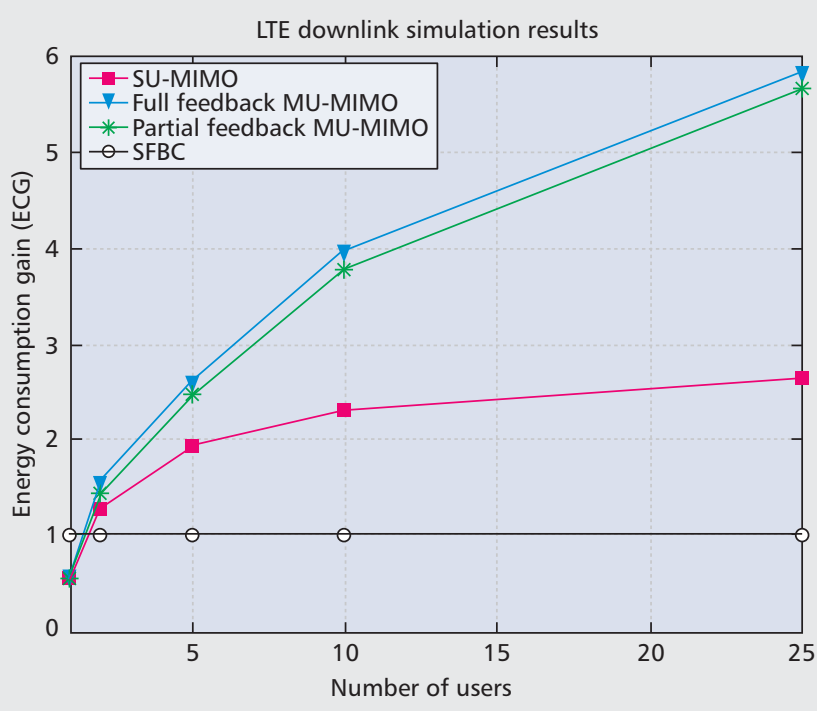

(b)

Figure 3. a) Simulated ECG of the frequency domain bandwidth expansion as a function of the required SNR at the receiver (after [5]); b) simulated ECG of various MIMO schemes, relative to SFBC all at $3 \mathrm{~b} / \mathrm{s} / \mathrm{Hz}$ spectral efficiency (after [6]).

QAM modulation (4 b/s/Hz maximum data rate) to be replaced by QPSK $(2 \mathrm{~b} / \mathrm{s} / \mathrm{Hz}$ maximum data rate), which would require a lower SINR for reliable operation. The results show that as the SINR increases, so does the potential improvement in ECG from using the bandwidth expansion technique. Increasing the value of $\alpha$ beyond four is shown to provide diminishing returns in terms of ECG, except at very high values of SINR where very spectrally efficient modulation schemes would be used.

When the traffic load is high, the base station may be transmitting data to many users simultaneously, possibly using MIMO techniques. In this case, it is usually possible to exploit multiuser diversity to increase the overall multi-user capacity achieved via an opportunistic resource scheduling and allocation strategy. This is where the scheduler assigns resources according to the users' instantaneous channel conditions in the time, frequency, or/and space domains. The performance gains can be translated to further energy reduction at the transmitter. A link adaptation approach is also taken into consideration to ensure the most energy saving transmission mode is employed within the allocated resource for a required QoS level. As an example from [7], Fig. 3b shows the ECG performance of different MIMO precoding schemes compared to using the single-user MIMO diversity scheme space frequency block coding (SFBC) as the baseline case. The multi-user MIMO schemes exploiting a higher degree of diversity achieve lower cost in terms of required transmitter energy for each information bit. When the number of mobile users is large enough, performance evaluation results show that a fivefold energy gain can be achieved by multi-user MIMO through employing appropriate link adaptation and resource scheduling approaches compared to an SFBC system.

Future work in this area will study the best combination of scheduling techniques from an energy efficiency perspective across the range of traffic loads experienced in future LTE networks.

\section{CASE Study 2:}

\section{InTERfEREnCE MANAgEMENT AND Mitigation}

Interference cancellation schemes are indispensable to combat interference in any practical communication systems where multiple base stations share the same spectrum. The impact of interference is more severe as users move closer to the boundary region between two cells, leading to significant SINR and hence data rate reduction. Most existing interference cancellation schemes have been designed to increase the spectral efficiency and data rate, while overlooking energy efficiency. However, research efforts in the Green Radio program are focused on developing energy-efficient interference cancellation schemes. If the level of interference can be reduced at mobile terminals, it will permit base stations to reduce the wireless transmission energy without compromising the SINR of the wireless link. There are two complementary strategies being considered, as shown in Fig. 4a: distributed antenna systems and receiver interference cancellation.

One way to reduce interference in cellular systems is to coordinate the multiple antennas of the adjacent base stations to form a distributed antenna system (DAS) [8]. For the resulting coordinating DAS, each and every cell edge user is collaboratively served by all of its surrounding base stations rather than only by the single best base station. This permits the interference to users on the cell edge to be effectively controlled and mitigated by coordinated transmit beamforming at all of the participating base stations. The following three schemes can be used by coordinating downlink beamforming: 


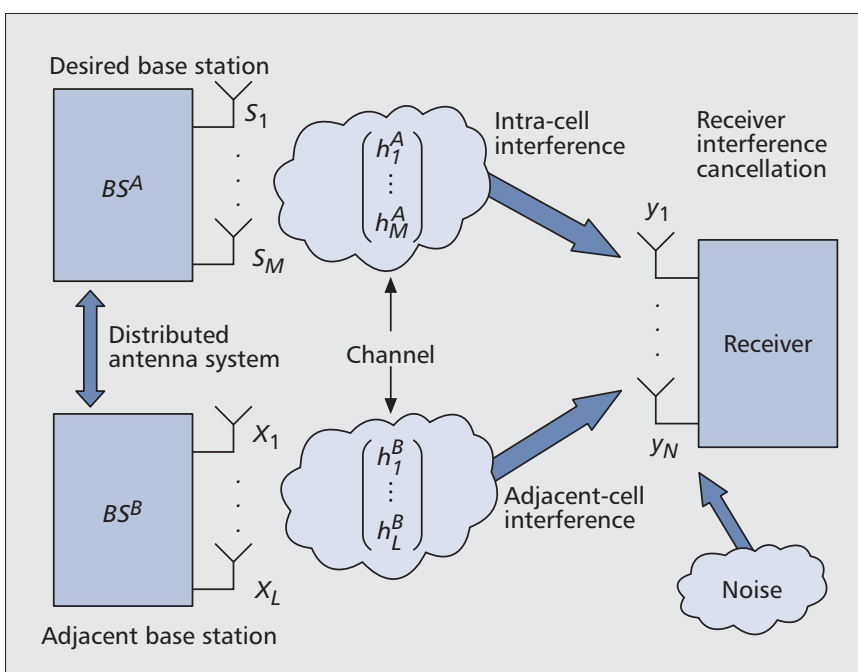

(a)

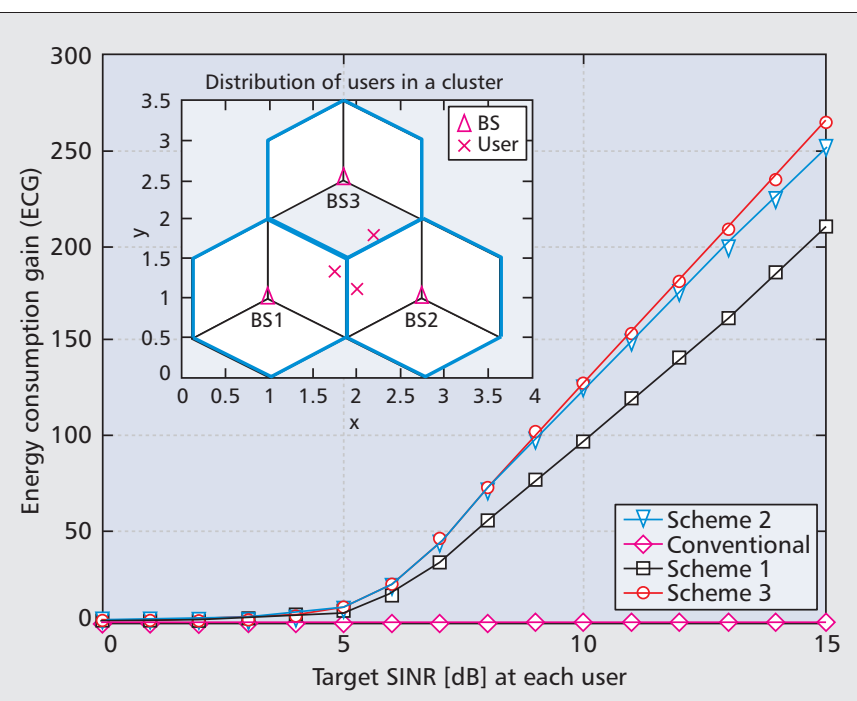

(b)

Figure 4. a) Example scenario for transmitter and/or receiver interference cancellation showing multiple base stations transmitting to a multiple antenna receiver; and b) performance comparison of three DAS schemes, plotting ECG (relative to no DAS case) vs SNR.

- The user is served by the base station providing the highest SINR while other base stations avoid transmitting signal energy toward that user.

- All users are served by multiple base stations using multiple antenna beamforming and coherent user-end combining (i.e., full exploitation of the interference suppression capability offered by the DAS).

- Users are allocated to one or more base stations based on their position.

These three schemes are compared in terms of ECG vs. SINR against the conventional noncooperative case in Fig. $4 b$ for a cluster of three cells with one user per cell. The results show that all three schemes significantly outperform the conventional system at high SINRs, with schemes 2 and 3 outperforming scheme 1. However, scheme 1 may be preferable over schemes $2 / 3$ in practical implementation, since it requires much less channel data about the users to be exchanged between the base stations and hence less energy consumption.

An alternative scheme to DAS is to apply interference cancellation techniques at a multiple-antenna receiver. The performance of different algorithms has been compared in [9] for different numbers of transmitting antennas. Linear zero forcing ( $\mathrm{ZF}$ ) and minimum mean squared error (MMSE) techniques have been compared, along with nonlinear successive interference cancellation (SIC) variants of these methods. Generally, it is observed that more transmission energy is required as the number of transmit antennas increases. This is expected as intracell interference increases with the number of transmit antennas, resulting in higher transmission energy to maintain the same SINR.

In the absence of co-channel interference from neighboring base stations, it is observed that the MMSE weight optimization approach provides better transmission energy savings than the ZF approach at the desired BS; with the SIC structure performing better than the linear receiver structure. This is because while the $\mathrm{ZF}$ criterion nulls out intracell interference but greatly amplifies adjacent-cell interference plus noise, the MMSE criterion jointly minimizes both intracell interference and noise, thus causing less severe amplification to the adjacent-cell interference and noise components. We also observe the same energy consumption trend when three adjacent base stations are present. The ECR values are around 3.4 times poorer than in the absence of co-channel interference for all receivers. This is because traditional interference cancellation (IC) techniques are often implemented at the link level (i.e., the point-topoint link between the desired BS and the receiver in this case). These link-level IC techniques are able to mitigate intracell interference but treat adjacent-cell interference simply as noise. More intelligent methods to cancel adjacent cell interference will be studied in future work, along with consideration of the most energy-efficient combination of IC techniques at both base stations and mobile terminals.

\section{CASE Study 3:}

\section{Energy-Efficient Routing AND MULtihop}

In a similar manner to the interference suppression techniques described above, the use of relays to exchange information between a base station and a mobile terminal may be an efficient way to improve base station energy efficiency. This is because the transmission distance can be reduced, increasing data rates or permitting reductions in transmission energy. Relays can enable important reductions of network energy consumption without complicated infrastructure modifications. These may be deployed in streets or buildings to provide improved signal quality to locations that might otherwise experience poor QoS.

In [10], the energy efficiency of several trans- 


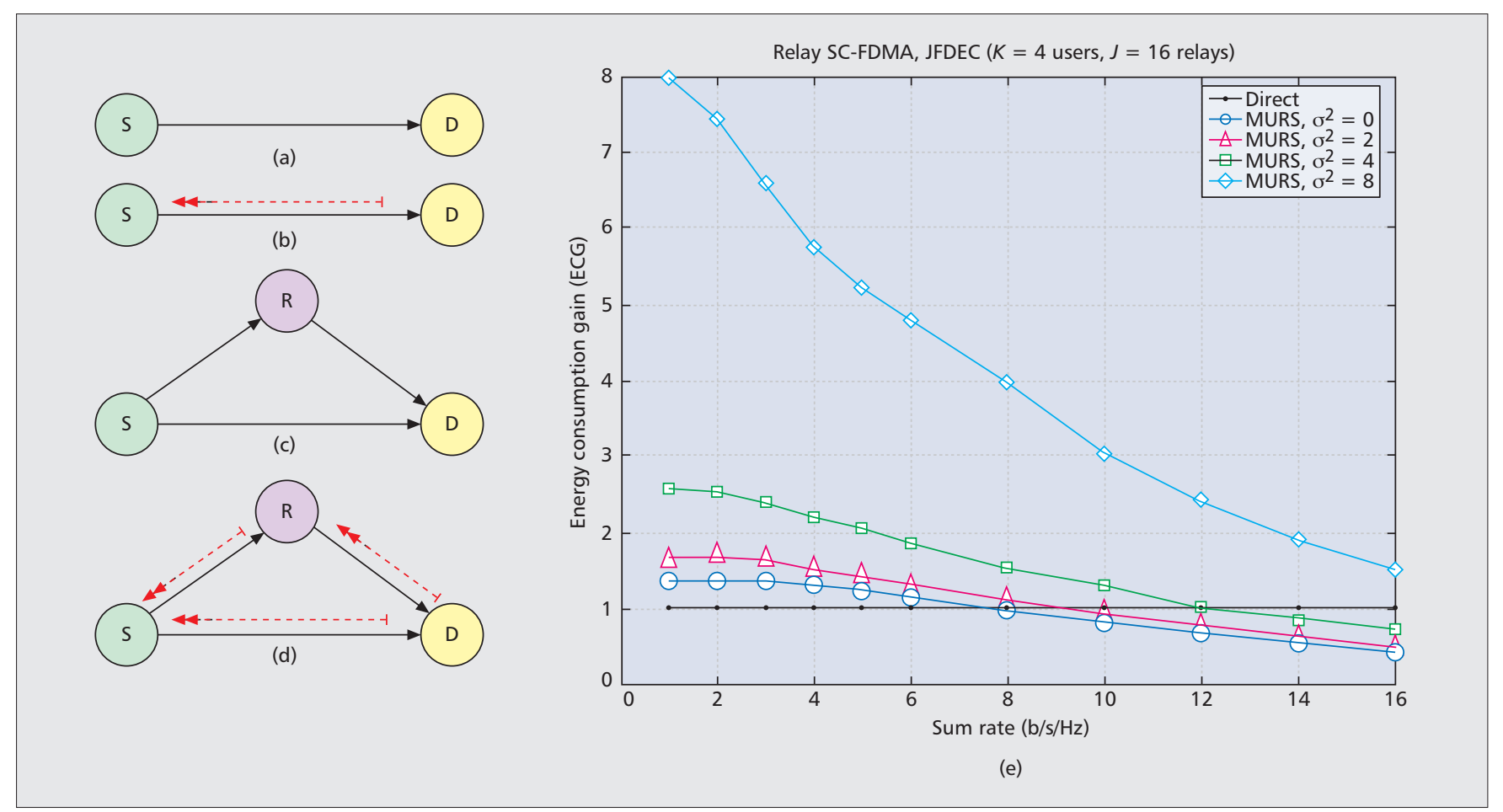

Figure 5. a) Direct wireless link with average channel knowledge; b) direct wireless link with instantaneous feedback of channel conditions; c) relay link with average channel knowledge; d) relay link with instantaneous feedback of channel conditions; e) performance gains of relay links after [11].

mission schemes shown in Figs. 5a-d are directly compared. Parts a and b show a conventional base station-mobile station link with average and instantaneous channel state feedback, respectively. Parts (c) and (d) show the case where a relay is present, again with average/instantaneous channel state feedback. It has been shown that the use of instantaneous channel feedback, which is the state of the art for resource allocation schemes, significantly reduces energy consumption compared to the case where only average channel state information is available. On the other hand, the impact of using a relay for communication is known to have a particularly strong impact for high signal-to-noise ratio (SNR) and low packet error rate conditions. This observation is in line with the basic conclusion from the literature that for fixed data rates, relaying is a particularly useful technique for high SNRs (or low packet error rates) because of the presence of the base station-relay-terminal path [11]; in this work, this conclusion is validated from an energy consumption perspective.

In [12], the energy efficiency of opportunistic cooperative relaying designed for the multiuser single-carrier frequency-division multiple access (SC-FDMA) uplink (mobile-to-base link) is investigated with the aid of a singlerelay amplify-and-forward (AF) scheme. The AF relay estimates the received power of each subband and equalizes the power differences of the subbands, which corresponds to subbandbased equalization. A joint frequency-domain equalization and combining (JFDEC) aided receiver is employed at the base station. In this scenario, there are 4 transmitting terminals and 16 available relays. The energy reduction of the proposed design is a direct benefit of spatial, frequency and selection diversity. In contrast to [11], where no terrain effects, termed shadowing, were considered, they are included in these results. In this case, the shadowing variance becomes an important parameter and expresses the variability in the environment due to buildings and other large obstacles. It may be observed in Fig. 5e that if the SNR is relatively low, the proposed multi-user relay selection (MU-RS) aided cooperative system provides an ECG of up to 8 relative to the no-relay "direct" case when experiencing a shadowing variance of 0-8 dB. However, as the operating SNR increases to a relatively high value and the target data rate increases correspondingly, the benefits of invoking an MU-RS cooperative system erode. This is not unexpected, because sharing the total transmit power between the source and relay as well as the provision of two time slots results in a throughput loss, which is not fully compensated by the relaying gain attained. It is anticipated that similar performance results will be observed for the downlink case as well.

One important future target for the work in this area is to be able to compare the energy efficiency of relay techniques with the use of femtocells. Relays provide a connection to the Internet through the nearest wireless base station. Conversely, femtocells are small low-power base stations installed in the home or office that use a wired Internet connection to provide service. Understanding the full impact of the energy consumption of these differing forms of network connection is an important but challenging task for the Green Radio project. 


\section{CONCLUSIONS}

This article has described the approach being taken in the Mobile VCE project to study novel approaches to reducing the energy consumption of wireless links, particularly in improving the design and operation of wireless base stations. Analysis has shown that when accounting for manufacturing or embodied energy costs, base stations have a much higher operational energy budget than mobile terminals. Proper modeling of the energy consumption of base stations has been shown to be an important issue when trying to obtain a clear view of how different radio technologies can reduce energy consumption. Three case studies of current research in resource allocation, interference suppression, and multihop routing have also been discussed. The means by which these methods can lead to energy savings have been described, and initial results that estimate the performance benefits of these techniques have been presented. The Green Radio project is a three-year program, which started in January 2009 and is starting to deliver initial results, some of which are described and discussed here. The project is being led by industry with the expectation that the most promising research outcomes can feed into future energy-efficient wireless standards and products.

\section{ACKNOWLEDGEMENTS}

The work reported has formed part of the Green Radio Core 5 Research Program of the Virtual Centre of Excellence in Mobile \& Personal Communications, http://www.mobilevce.com. This research is funded by the industrial companies who are members of Mobile VCE and by the U.K. EPSRC under grant numbers EP/G060584/1, EP/G06041X/1, EP/G062420/1, and $\mathrm{EP} / \mathrm{G} 064105 / 1$.

The authors express their grateful thanks to the industrial leadership team, including Simon Fletcher (NEC), David Lister (Vodafone), Terence Dodgson (Nokia-Siemens Networks), and Andy Jeffries (Nortel).

\section{REFERENCES}

[1] T. Edler, "Green Base Stations - How to Minimize $\mathrm{CO}_{2}$ Emission in Operator Networks," Ericsson seminar, Bath Base Station Conf., 2008.

[2] H. Holma and A. Toskala, LTE for UMTS, Wiley, 2009.

[3] G. Fischer, "Next-Generation Base Station Radio Frequency Architecture," Bell Labs Tech. J., vol. 12, no. 2, 2007, pp. 3-18.

[4] K. Bumman, M. Junghwan, and K. Ildu, "Efficiently Amplified," IEEE Microwave Mag., vol. 11, no. 5, Aug. 2010, pp. 87-100.

[5] P. Wright et al., "A Methodology for Realizing High Efficiency Class-J in A Linear and Broadband PA," IEEE Trans. Microwave Theory and Techniques, vol. 57, 2009, pp. 3196-3204.

[6] S. Videv, H. Haas, and P. M. Grant, "Bandwidth-Energy Efficiency Trade-off with Variable Load in LTE," to be submitted to IEEE VTC Spring, Budapest, Hungary, 2011.

[7] K. C. Beh et al., "Power Efficient MIMO Techniques for 3GPP LTE and Beyond," Proc. IEEEVTC Fall, Anchorage, AK, Sept. 2009.

[8] T. A. Le and M. R. Nakhai, "'Throughput Analysis of Network Coding Enable Wireless Backhauls," submitted to IET Commun., 2010.

[9] I. Ku, C.-X. Wang, and P. M. Grant, "Impact of Receiver Interference Cancellation Techniques on Base Station Transmission Energy in MIMO Systems," submitted to IEEE ICC, Kyoto, Japan, 2011.
[10] I. Krikidis, J. S. Thompson, and P. M. Grant, "Cooperative Relaying with Feedback for Lifetime Maximization," Proc. IEEE ICC 2010 Wksp. E2NETS, Cape Town, South Africa, May 2010.

[11] J. N. Laneman, D. N. C. Tse, and G. W. Wornell, "Cooperative Diversity in Wireless Networks: Efficient Protocols and Outage Behavior," IEEE Trans. Info. Theory, vol. 50, no. 12, Dec. 2004, pp. 3062-80.

[12] J. Zhang, L.-L. Yang, and L. Hanzo, "Power-Efficient Opportunistic Amplify-and-Forward Single-Relay Aided Multi-User SC-FDMA Uplink," Proc. IEEE VTC Spring, Taipei, Taiwan, May 2010.

\section{BIOGRAPHIES}

CONGZHENG HAN received her B.Eng. and Ph.D. degrees in electronic and communications engineering from the University of Bristol, United Kingdom, in 2004 and 2008, respectively. She is currently a member of research staff in the Centre for Communications Research at the University of Bristol and is contributing to the Mobile VCE's Green Radio project. Her research interests include wireless LANs, future-generation standards (LTE), and power-efficient communications.

TIM HARROLD is a research fellow in the Centre for Communications Research, University of Bristol. He has worked on a wide variety of wireless projects in areas including indoor/outdoor propagation, self-organising ad hoc networks, smart antennas, ultra-wideband communications, and cognitive radio. This work has been supported by organizations including the UK's Mobile VCE, BT, ERA Technology, and Kyocera. He is currently on secondment to Toshiba Research Europe's Telecommunications Research Laboratory.

IOANNIS KRIKIDIS received his diploma from the Computer Engineering and Informatics Department (CEID) of the University of Patras, Greece, in 2000, and M.Sc. and Ph.D. degrees from Ecole Nationale Supérieure des Télécommunications (ENST), Paris, France, in 2001 and 2005, respectively. From 2007 to 2010 he was a research fellow in the School of Engineering and Electronics at the University of Edinburgh, United Kingdom. He has now been elected an assistant professor at CEID.

IVAN KU received his B.Eng. degree in electronics engineering and M.Eng.Sc. degree in communications from Multimedia University (MMU), Malaysia, in 2001 and 2006, respectively. Since April 2009 he has been a Ph.D. student at Heriot-Watt University, Edinburgh, United Kingdom. His research interests include cooperative communication, multiuser detection theory, and digital signal processing.

TUAN ANH LE received his B.Eng. and M.Sc. degrees from Hanoi University of Technology, Vietnam, in 2002 and 2004 , respectively. Both are in electronics and telecommunications. He is currently a Ph.D. research student in the Centre for Telecommunications Research, King's College London, and a researcher of the Mobile VCE Core 5 Research Program on Green Radio. His interests are multicell processing, multicell beamforming, and convex optimization.

STEFAN VIDEV got his first two degrees from Jacobs University Bremen (B.Sc. in electrical engineering and computer science, M.Sc. in communication, systems, and electronics). He is currently a Ph.D. student at the Institute for Digital Communications at the University of Edinburgh. His research interests are in cognitive radio, green radio, resource allocation, and interference avoidance.

JIAYI ZHANG received his B.Eng. degree from Nanjing University of Aeronautics and Astronautics, China, and his M.Sc. degree from the University of Southampton, United Kingdom, in 2006 and 2007, respectively. He is currently continuing his study toward a Ph.D. degree within the School of Electronics and Computer Science at the University of Southampton. His research interests include SC-FDMA, MIMO-OFDM, frequency-domain equalization, turbo detection, transmitter preprocessing, cooperative relaying, multicell processing, and cross-layer optimization.

SIMON ARMOUR is a senior lecturer at the University of Bristol. He has led collaborative projects with a number of industrial partners and now leads Bristol's involvement in the MVCE's Green Radio project. His work has resulted in
The Green Radio

project is a three-

year program, which

started in January

2009 and is starting

to deliver initial

results. The project is being led by industry with the expectation

that the most promising research outcomes can feed into future energyefficient wireless

standards and products. 
approximately 100 papers published in international journals and conferences and 11 patents. The majority of the latter are now owned by industry.

Peter M. GRANT [F] is currently a senior professorial research fellow at the University of Edinburgh. He was in 2007 appointed to be the 8th Regius Professor of Engineering at the University of Edinburgh. In 2009 he was made an officer of the Order of the British Empire (OBE) in the Queen's birthday honors list. He holds fellowships of the IET, Royal Academy of Engineering, and Royal Society of Edinburgh.

HARALD HAAS received his Ph.D. degree from the University of Edinburgh in 2001. From 2001 to 2002 he was a research project manager at Siemens in Munich. He joined Jacobs University Bremen in 2002 as associate professor before returning to the University of Edinburgh where he holds a personal Chair in Mobile Communications. His main research interests are decentralized interference management in wireless networks, multiple antenna concepts, and optical wireless communication.

LAJOS HANZO [F] received his Master's degree in electronics in 1976 and his doctorate in 1983 from the Technical University of Budapest. In 2010 he was awarded the university's highest honor, the Doctor Honaris Causa. He is also an IEEE Distinguished Lecturer of both the Communications and Vehicular Societies as well as a Fellow of the IEE/IET and Royal Academy of Engineering.
Mohammad Reza NAKHAl received his Ph.D. degree in electronic engineering from King's College, University of London in 2000. He worked as a postdoctoral research fellow in the Centre for Communication Systems Research, University of Surrey. Since 2001 he has been an academic member of staff in the Centre for Telecommunications Research, King's College London. His current research interests include cognitive radio, cooperative communications, optimization, and communications theory.

JOHN THOMPSON (john.thompson@ed.ac.uk) is currently a reader at the School of Engineering at the University of Edinburgh. He currently leads the Green Radio activities at the university. He has published over 160 journal and conference papers to date. His research interests are in signal processing, multiple antenna communications, multihop techniques, and green communications. He was Technical Program Co-Chair for IEEE GLOBECOM 2010.

Cheng-XIANg WANG received his Ph.D. degree from Aalborg University, Denmark, in 2004. He joined Heriot-Watt University as a lecturer in 2005 and became a reader in 2009. His research interests include wireless channel modeling, cognitive radio networks, vehicular communication networks, green communications, and (beyond) 4G. He has published one book chapter and over 130 papers in journals and conferences. He received the IEEE GLOBECOM Best Paper Award in 2010. 